\title{
Assistência de enfermagem ao laringectomizado no período pós-operatório
}

\author{
Laryngectomized in postoperative period: the nursing care
}

Luciana de Souza Lima da Silva, ${ }^{1}$ Maria Helena Pinto² e Márcia M Fontão Zago ${ }^{3}$

\begin{abstract}
Resumo
O s objetivos deste estudo foram identificar os diagnósticos de enfermagem no período pósoperatório tardio de laringectomia total e traçar um plano de intervenções de enfermagem para os diagnósticos identificados. Participaram deste estudo 10 pacientes submetidos a laringectomia total, após o tratamento radioterápico, com tempo de pós-operatório de 3 a 18 meses. A coleta de dados foi realizada por meio de entrevista e exame físico, algumas vezes com a presença de familiares que complementavam as informações. Foram identificados 16 diagnósticos de enfermagem segundo a taxonomia de N AN D A (2000). As intervenções foram descritas para cada diagnóstico conforme sugestão de Carpenito (1999) e a experiência das autoras. Este estudo demonstrou que o diagnóstico de enfermagem é o guia para uma assistência de enfermagem efetiva, deve ser utilizado para direcionar as ações de enfermagem, quer seja durante a internação hospitalar como durante o seguimento ambulatorial, enfatizando que a assistência ao laringectomizado deve ser voltada não só para o aspecto biológico mas também para o social e 0 psicológico, pois é um paciente que retorna ao convívio familiar com bruscas alterações de estilo de vida, e muitas vezes altera também todo o contexto familiar.
\end{abstract}

Palavras-chave: laringectomia; pacientes; diagnóstico de enfermagem; cuidados de enfermagem; cuidados pós-operatórios.

${ }^{1}$ Acadêmica do Curso de Graduação em Enfermagem - Bolsista de iniciação científica FAM ERP.

Enviar correspondência para L.S.L.S. Rua Abdo M uanis 1101, apto 62; 15.090-140 São José do Rio Preto, SP - Brasil.

${ }^{2}$ Enfermeira. D outoranda em Enfermagem Fundamental da EERP/U SP e D ocente da FAM ERP.

${ }^{3}$ Enfermeira. Professora associada do DEGE-EERP/USP.

Recebido em junho de 2001. 


\begin{abstract}
This study ai med at identify the nursing diagnoses of completly laringectomized patients in the late postoperative period and to outline a nursing approach in relation to these diagnoses. Ten laryngectomized patient were inclued in the study, the postoperative period; after the radioterapy treatment was between 3 and 18 months. D ata were collected by means of a interviews and physical examinations. Sometimes these were made with the presence of the patient's relatives with the purpose of gathering more information about him/her. Sixteen nursing diagnosis were identified according to N AN D A taxonomy. For each diagnosis, interventions based on Carpenitos' suggestion as well as on the authors' experience were used. This study showed that the nursing diagnosis can be the starting point for the nursing procedures either during the in-and-out patient's follow- ups. The nursing assistance his/her biological features, but also to the social and familial enviroment. This patient has returned home with abrupt alterations on his/her lifestyle, and consequently, most of the time chaging the familial enviroment.
\end{abstract}

Key words: laryngectomy; patients, nursing diagnosis; nursing care; postoperative care.

\section{INTRODUÇÃO}

0 câncer de laringe é um dos mais comuns a atingir a região da cabeça e pescoço, representando cerca de $25 \%$ dos tumores malignos que acometem esta área e $2 \%$ de todas as doenças malignas. A sua incidência é maior nos homens do que nas mulheres e atinge predominantemente a faixa etária acima de 50 anos de idade. ${ }^{1}$

Anatomicamente, a laringe está situada na região infra-hióidea, abaixo da faringe e acima da traquéia. Fisiologicamente desempenha a função respiratória, esfincteriana (protegendo as vias aéreas inferiores contra a penetração de corpos estranhos e alimentos) e fonatória (pela vibração das cordas vocais). ${ }^{2}$

As pessoas com câncer de laringe, em estágio avançado submetem-se ao procedimento cirúrgico denominado "laringectomia total", que é a remoção da laringe, da cartilagem tireóide, pregas vocais, epiglote e anexos. A parte superior da faringe é suturada à base da língua e a traquéia é suturada à pele da base do pescoço, dando origem a um traqueostoma, para preservar a função respiratória. Após a cirurgia é comum 0 tratamento radioterápico. ${ }^{2-4}$

Portanto, a laringectomia total é uma cirurgia que produz alterações fisiológicas que incluem alteração da via respiratória, traqueostomia permanente, afonia, diminuição da atividade motora do ombro, braço e pescoço e diminuição do olfato e paladar; e alterações psicossociais que abrangem a imagem corporal, a comunicação verbal-oral, as atividades sociais e a autoestima da pessoa. ${ }^{5,6}$

Para que o planejamento da assistência de enfermagem seja adequado e individualizado é preciso utilizar-se do processo de enfermagem. Para Christensen e Kenney ${ }^{7} 0$ processo de enfermagem é uma atividade deliberada, na qual a prática de enfermagem é realizada de maneira sistematizada, lógica e racional, utilizando um conhecimento compreensivo e essencial para avaliar o estado de saúde do cliente e assim, prestar uma assistência de enfermagem de qualidade. É uma abordagem de solução de problemas para a enfermagem, uma prática que tem 0 propósito de resolver, reduzir ou prevenir problemas de saúde e/ou promover a adaptação do cliente a esses problemas. ${ }^{8,9}$

Portanto, o processo de enfermagem é um método sistemático e planejado pelo qual a assistência deixa de ser puramente intuitiva e passa a ter caráter científico, e foi o que levou ao reconhecimento da profissão enfermagem nos anos sessenta. ${ }^{10}$

0 processo de enfermagem consiste de cinco fases ou passos: coleta de dados, diagnóstico de enfermagem, planejamento, implementação e avaliação, que na realidade estão inter-relacionados e formam um ciclo contínuo de reflexão e ação. ${ }^{8-10}$ 
0 diagnóstico de enfermagem é definido pela N orth American N ursing Diagnosis Association (N AN D A) como o julgamento clínico das respostas do indivíduo, da família ou da comunidade a processos vitais ou a problemas de saúde reais ou potenciais, os quais fornecem a base para a seleção das intervenções de enfermagem para atingir resultados pelos quais a enfermeira é responsável. ${ }^{11}$

A fase do diagnóstico de enfermagem é considerada não apenas como uma simples listagem de problemas, mas sim uma fase que envolve análise, interpretação dos dados coletados, avaliação crítica e tomada de decisão. ${ }^{10}$

Risner ${ }^{12}$ afirma que a diferença entre 0 diagnóstico médico e diagnóstico de enfermagem é que o primeiro identifica e rotula doenças e o segundo descreve os efeitos destes sintomas e condições patológicas nas atividades e, no sentido da vida do cliente, é a afirmação das respostas do cliente a uma condição ou situação.

A utilização da taxonomia de diagnóstico de enfermagem (NANDA) contribui para uniformizar a linguagem dos enfermeiros, melhorar a comunicação, fornecer dados para pesquisa e assim desenvolver um corpo de conhecimentos para a profissão. ${ }^{13}$ Para a organização dos diagnósticos de enfermagem a N AN D A utilizou como estrutura conceitual os chamados "nove padrões de respostas humanas" que são: trocar, comunicar, relacionar, valorizar, escolher, mover, perceber, conhecer e sentir.

A fase do diagnóstico de enfermagem merece destaque pois é um guia para as próximas etapas: o planejamento, implementação e avaliação da assistência de enfermagem.

Considerando que o enfermeiro inclui 0 preparo para a alta hospitalar no planejamento da assistência de todo paciente submetido a laringectomia total, é importante lembrar da necessidade de uma avaliação pós alta, tendo em vista a resposta humana às transformações conseqüentes da laringectomia.

Avaliação é um processo contínuo e sistemático de comparação das respostas do cliente com suas metas desejadas, que determina o nível de progresso do cliente e revisa o plano de cuidado. ${ }^{8}$
Cabe ao enfermeiro acompanhar 0 paciente nos retornos médicos com o objetivo de avaliar a condição do paciente tanto física como psicossocial e se necessário elaborar novas intervenções juntamente com o paciente e seus familiares.

Frente a estas considerações, este estudo teve os seguintes objetivos:

a) identificar os diagnósticos de enfermagem no período pós alta hospitalar (pós-operatório tardio) de laringectomia total;

b) traçar um plano de intervenções frente aos diagnósticos deenfermagem identificados.

\section{MATERIAL E MÉTODOS}

0 presente estudo foi desenvolvido no ambulatório de otorrinolaringologia do $\mathrm{H}$ ospital de Base de São José do Rio Preto, após a aprovação do projeto de pesquisa pelo Comitê de Ética em Pesquisa da Faculdade de M edicina de São José do Rio Preto.

A amostra deste estudo foi constituída de 10 pacientes laringectomizados com tempo de pós-operatório de três meses a 18 meses. Participaram deste estudo os pacientes que concordaram em participar da investigação, com a assinatura prévia do termo de consentimento esclarecido. 0 s acompanhantes assinaram pelos pacientes que não sabiam escrever. Todos os pacientes já haviam feito a radioterapia.

A coleta de dados foi realizada por meio de uma entrevista e exame físico, seguindo um instrumento de coleta de dados, previamente construído de acordo com 0 modelo eclético citado por Christensen e Kenney. ${ }^{7}$ A maioria dos pacientes estavam acompanhados pelas esposas ou filhos, que muitas vezes complementavam as informações, facilitando assim a coleta de dados.

As entrevistas foram realizadas nos dias que os pacientes estavam agendados para a consulta com a fonoaudióloga ou após a reunião mensal realizada com os pacientes laringectomizados, alguns familiares, a fonoaudióloga e os autores, no período de julho a novembro de 2000.

0 instrumento para a coleta de dados foi elaborado considerando a situação clínica da 
população em estudo, bem como a natureza das informações que se espera obter (anexo1), pois em pacientes que compartilham a mesma clínica existe uma probabilidade maior de surgirem necessidades de cuidados comuns, o que deve ser considerado como fator norteador para o desenvolvimento da coleta de dados.

Para atingir o segundo objetivo foram traçadas as intervenções a cada diagnóstico, segundo as sugestões de $\mathrm{C}$ arpenito. ${ }^{14} \mathrm{~A}$ autora afirma que as intervenções de enfermagem são aquelas ações que 0 enfermeiro pode ordenar legalmente, que sejam implementadas pela equipe, com o objetivo de tratar, prevenir e monitorar os diagnósticos.

Vale ressaltar que a população do estudo foi constituída de 10 pacientes póslaringectomia total, sem esvaziamento cervical, com idade entre 48 e 80 anos (média de
64 anos); todos do sexo masculino; (80\%) casado; (20\%) viúvo; (30\%) ainda faz uso de bebida alcoólica e (80\%) ex-tabagista; (80\%) nível fundamental de escolaridade incompleto e $(20 \%)$ de analfabetos; $80 \%$ aposentados e religião predominantemente católica. Como já foi dito, todos os pacientes foram entrevistados apóso tratamento radioterápico.

\section{RESULTADOS E DISCUSSÃO}

$\mathrm{N}$ a análise dos dados, em relação ao primeiro objetivo, elaborou-se 16 diagnósticos de enfermagem para os 10 pacientes participantes do estudo. As informações referentes aos tipos de diagnósticos de enfermagem, distribuídos em cada um dos padrões de resposta humana encontrados nesta pesquisa, apresentam-se na Tabela 1.

Tabela 1. Distribuição de Diagnósticos de Enfermagem (DE) e de padrões de respostas humanas, segundo a Taxonomia de NANDA em laringectomizados, no pós-operatório tárdio em número absoluto e porcentagem

\begin{tabular}{|c|c|c|c|}
\hline \multirow{2}{*}{ Padrão de resposta } & \multirow{2}{*}{ Diagnóstico de enfermagem } & \multicolumn{2}{|c|}{ Pacientes } \\
\hline & & $n^{\circ}$ & $\%$ \\
\hline \multirow{4}{*}{ Trocar } & Integridade tissular prejudicada & 8 & $\overline{80,0}$ \\
\hline & Mucosa oral alterada & 2 & 20,0 \\
\hline & Deglutição prejudicada & 2 & 20,0 \\
\hline & Risco para aspiração & 2 & 20,0 \\
\hline Comunicar & Comunicação verbal prejudicada & 10 & 100,0 \\
\hline \multirow{2}{*}{ Relacionar } & Processo familiar alterado & 1 & 10,0 \\
\hline & Interação social prejudicada & 3 & 30,0 \\
\hline Valorizar & Angústia espiritual & 1 & 10,0 \\
\hline \multirow{2}{*}{ Escolher } & Adaptação prejudicada & 3 & 30,0 \\
\hline & Enfrentamento individual prejudicado & 3 & 30,0 \\
\hline Mover & Distúrbio no padrão do sono & 2 & 20,0 \\
\hline \multirow{3}{*}{ Perceber } & Alteração sensorial de percepção: olfato & 6 & 60,0 \\
\hline & Baixo auto estima situacional & 3 & 30,0 \\
\hline & Distúrbio da imagem corporal & 3 & 30,0 \\
\hline Conhecer & Déficit de conhecimento sobre a cirurgia & 1 & 10,0 \\
\hline Sentir & Dor na região cervical & 3 & 30,0 \\
\hline
\end{tabular}

Em relação aos diagnósticos de enfermagem (DE) do padrão trocar, a integridade tissular prejudicada está relacionada ao tratamento radioterápico, caracterizado pelo edema, hiperemia e endurecimento da região cervical. 0 tratamento radioterápico gera uma certa toxicidade na região a ser irradiada, nesta região é comum encontrarmos alteração da pele e mucosa oral além da perda do paladar e diminuição da saliva. ${ }^{15}$

0 diagnóstico de mucosa oral alterada está relacionado ao tratamento radioterápico caracterizado pela estomatite, assim como o DE de deglutição prejudicada está relacionado à estomatite secundária, à radioterapia e caracterizado pelo relato do paciente de dificuldade para comer.

O diagnóstico de risco para aspiração foi atribuído a dois pacientes e está 
relacionado a uma complicação da laringectomia, à fistula traqueo-esofágica, que leva o paciente a permanecer com sonda nasogástrica / nasoentérica para alimentação e com a cânula de traqueostomia com cuff insuflado até a cicatrização da fístula.

0 início da formação da fístula ocorre com a deiscência de sutura da mucosa oral ou faríngea e pode estar associada a infecção cirúrgica e infecção pulmonar, devido a aspiração da saliva. ${ }^{16}$

O DE do padrão comunicar, comunicação verbal prejudicada, todos os pacientes apresentam devido ao procedimento cirúrgico, no qual são removidas as estruturas que produzem 0 som laríngeo. ${ }^{6}$

A afonia e a alteração da imagem corporal modificam o modo de vida da pessoa causando problemas tanto psicológicos como sociais, como percebemos no padrão de resposta humana relacionar, com os dois $D E$ encontrados: processo familiar alterado e a interação social alterada.

O DE processo familiar alterado está relacionado à mudança de estado de saúde $\mathrm{e}$ à barreira da comunicação e está evidenciado pela mudança na participação na tomada de decisão e o DE interação social alterada está relacionado à presença do traqueostoma e à afonia, evidenciado pela resistência em participar de eventos como missa ou festas de aniversário, os dois pacientes que trabalham são trabalhadores rurais, que verbalizam gostar desta ocupação, porqueficam sozinhos, longe de todos (estas informações foram dadas pelos acompanhantes e confirmadas pelo paciente). Segundo W hite et $\mathrm{a}^{17}$ as mudanças causadas por uma doença ou cirurgia alteram o estado psicológico e consequentemente as atividades sociais.

Zago et $a^{6}$ afirma que a alteração da imagem corporal e a alteração da comunicação levam alguns pacientes ao isolamento social e também a desenvolverem uma certa dependência prolongada de seus familiares, caracterizada pela mudança na participação na tomada de decisão em família. Segundo o Instituto $\mathrm{N}$ acional de Câncer, ${ }^{1}$ 50\% dos pacientes laringectomizado ficam reclusos em suas residências longe do convívio social.

D entro do padrão valorizar foi encontrado em um paciente o DE - angústia espiritual, que está relacionado a conflito entre crenças, regime de saúde prescrito e sistema de valores desafiados, caracterizado por questionamentos da própria existência e manifestação de sensação de solidão, abandono e raiva quando questionado sobre as necessidades espirituais.

Segundo $C$ arpenito ${ }^{14}$ a angústia espiritual é um estado em que o indivíduo está em risco para apresentar um distúrbio no sistema de valores que proporciona força, esperança e significado para a vida.

0 bem estar espiritual pode ser descrito como a harmonia e a interconexão de relacionamentos. Esses relacionamentos podem usar a sua espiritualidade para superar 0 estágio de desespero e integridade. ${ }^{14}$ Vale ressaltar que este paciente sempre comparece aos retornos sozinho e está sendo acompanhado pela psicóloga.

No padrão escolher foram identificados dois DE: adaptação prejudicada, que está relacionado a mudanças do estado de saúde que requer mudança de estilo de vida evidenciado pelo uso abusivo de bebidas alcoólicas, mesmo após as orientações sobre os riscos à recidiva da doença; e o enfrentamento individual prejudicado. relacionado a falta de suporte social por membros da família, evidenciado pelo comportamento destrutivo em relação a si próprio e a falta de interesse e empenho da família na reabilitação do paciente.

O DE distúrbio no padrão do sono, que pertence ao padrão de resposta humana mover foi identificado em dois pacientes e está relacionado a posição de repouso, cabeceira não el evada e estase de secreção pulmonar, evidenciado por verbalização do paciente de aumento da tosse na posição deitada.

No padrão perceber o $\mathrm{DE}$ alteração sensorial de percepção: olfato, está relacionado à laringectomia. As alterações no olfato e no paladar ocorrem após a laringectomia total porque o paciente passa a respirar diretamente pela traquéia, 0 ar não passa através do nariz para os órgãos olfativos terminais. Como 0 olfato e o paladar estão intimamente ligados, suas sensações de paladar são alteradas. $M$ as, com o passar do tempo, o paciente comumente se acomoda a este problema, o que justifica que nem todos os pacientes referiram a alteração do olfato. ${ }^{15}$ 
O DE baixo auto estima situacional, embora os fatores relacionados ainda não tenham sido desenvolvidos pela NANDA, parece estar relacionado a incapacidade de enfrentar ou de aceitar as mudanças tanto de imagem corporal como de estilo de vida, evidenciado pela dificuldade em tomar decisões frente a situação de ser laringectomizado, passando as decisões para outro membro da família. I sto foi evidenciado em três pacientes.

O distúrbio da imagem corporal é um $\mathrm{DE}$ que está relacionado à cirurgia de laringectomia evidenciado pela "verbalização" de percepções alteradas da própria aparência devido ao traqueostoma.

N o padrão conhecer O $D E$ - déficit de conhecimento sobre as conseqüências da laringectomia, foi encontrado somenteem um paciente que já era traqueostomizado antes da cirurgia, relacionado a falta de informação pré e pós-operatória evidenciado por questionamentos sobre a retirada da traqueostomia e a possibilidade de voltar a se comunicar oralmente como antes da cirurgia.

D ada essa complexa reestruturação do estilo de vida destes indivíduos a orientação pré-operatória e pós-operatória imediata se faz fundamental. 0 processo de reabilitação depende da compreensão e conseqüente aceitação do indivíduo quanto a seu estilo de vida atual. ${ }^{4}$

No padrão sentir o DE dor está relacionado a recidiva do tumor como complicação pós operatória em um paciente em dois ao tratamento radioterápico, evidenciado por verbalização do paciente e observação da expressão facial de dor.

$\mathrm{N}$ o que se refere ao segundo objetivo (traçar um plano de intervenções de enfermagem frente a esses diagnósticos) elaboramos as intervenções segundo Carpenito ${ }^{14}$ e as experiências das autoras.

Toda ação é realizada com algum objetivo, espera-se um resultado. Q uando um cliente não está progredindo na obtenção dos resultados esperados, o enfermeiro deve reavaliar a situação e modificar o plano de cuidados.

É importante ressaltar que as intervenções de enfermagem procedem tanto da prescrição do enfermeiro como do médico. As complicações pós-operatórias, como a dor à deglutição, a fístula e a recidiva tumoral são denominados por Carpenito ${ }^{14}$ problemas colaborativos. O s problemas colaborativos são certas complicações fisiológicas que os enfermeiros monitoram para detectar 0 estabelecimento ou a modificação subseqüente em seu estado, as intervenções são prescritas pelo médico e o enfermeiro monitora e avalia as condições do cliente e implementa as ordens prescritas pelo médico.

A diferença é que, para os diagnósticos de enfermagem, 0 enfermeiro prescreve 0 tratamento definitivo, ele pode evitar o risco de complicação; para os problemas colaborativos, entretanto, a prescrição do tratamento definitivo procede do médico e 0 enfermeiro colabora com o tratamento. Ela não pode evitar o risco da complicação fisiológica e legalmente não pode ordenar intervenções, ela prescreve e implementa intervenções do âmbito da enfermagem. ${ }^{14}$

U m paciente com fístula tem um problema colaborativo que é a complicação potencial: respiratória, que descreve a pessoa apresentando, ou em alto risco para apresentar, vários problemas respiratórios; neste caso a intervenção de enfermagem é o monitoramento do estado respiratório para detectar e diagnosticar o funcionamento anormal e comunicar ao médico.

O utro problema colaborativo, segundo Carpenito ${ }^{14}$ é a complicação potencial: equilíbrio negativo de nitrogênio; que descreve a pessoa apresentando, ou em risco para apresentar, catabolismo, quando é excretado mais nitrogênio da transformação do tecido do que é reposto pela ingesta. Este problema é atribuído ao paciente que apresenta dor a deglutição e recidiva tumoral. A intervenção é monitorar a aceitação alimentar e orientar a confecção de uma dieta balanceada para as suas necessidades corpóreas juntamente com o nutricionista e 0 médico.

As intervenções de enfermagem segundo os diagnósticos de enfermagem são apresentadas na Tabela 2. 
Tabela 2. Intervenções de enfermagem segundo os diagnósticos de enfermagem atribuídos aos pacientes laringectomizados no pós-operatório tardio

\begin{tabular}{|c|c|c|}
\hline $\begin{array}{l}\text { Diagnóstico de } \\
\text { enfermagem }\end{array}$ & Intervenções de enfermagem & Resultados esperados \\
\hline $\begin{array}{l}\text { Integridade } \\
\text { Tissular } \\
\text { Prejudicada }\end{array}$ & $\begin{array}{l}\text { Lavar a pele da região do pescoço somente com água e se } \\
\text { possível sabão neutro; } \\
\text { Orientar o uso de hidratante na região do pescoço; } \\
\text { Proteger a área lesada da luz do sol. }\end{array}$ & $\begin{array}{l}\text { O paciente deverá: } \\
\text { - participar do cuidado com o } \\
\text { tecido lesado; } \\
\text { - manifestar a melhora } \\
\text { progressiva da área lesada. }\end{array}$ \\
\hline $\begin{array}{l}\text { Mucosa oral } \\
\text { alterada }\end{array}$ & $\begin{array}{l}\text { Ensinar o paciente a fazer higiene oral com a solução oral } \\
\text { conforme prescrição médica; } \\
\text { Ensinar o paciente a alimentar-se com alimentos frios e } \\
\text { macios. }\end{array}$ & $\begin{array}{l}\text { O paciente deverá: } \\
\text { - apresentar integridade da } \\
\text { cavidade oral. }\end{array}$ \\
\hline $\begin{array}{l}\text { Deglutição } \\
\text { prejudicada }\end{array}$ & $\begin{array}{l}\text { Ensinar o paciente a fazer uso do analgésico prescrito pelo } \\
\text { médico antes das refeições; } \\
\text { Ensinar o paciente a alimentar-se lentamente com } \\
\text { alimentos líquidos sem excesso na temperatura fria ou } \\
\text { quente }\end{array}$ & $\begin{array}{l}\text { O paciente deverá: } \\
\text { - comunicar melhor } \\
\text { capacidade de deglutição. }\end{array}$ \\
\hline $\begin{array}{l}\text { Risco para } \\
\text { aspiração }\end{array}$ & $\begin{array}{l}\text { Manter o cuff da cânula de traqueostomia inflado; } \\
\text { Ensinar o paciente a sentar ou elevar a cabeceira para } \\
\text { administrar a alimentação pela sonda nasogátrica (SNG); } \\
\text { Ensinar o paciente ou o familiar a observar o } \\
\text { posicionamento da SNG antes da administração da } \\
\text { alimentação. }\end{array}$ & $\begin{array}{l}\text { O paciente deverá: } \\
\text { - não apresentar } \\
\text { aspiraçãa relatar medidas para } \\
\text { prevenção de aspiração. }\end{array}$ \\
\hline $\begin{array}{l}\text { Diagnóstico de } \\
\text { enfermagem }\end{array}$ & Intervenções de enfermagem & Resultados esperados \\
\hline $\begin{array}{l}\text { Comunicação } \\
\text { verbal } \\
\text { prejudicada }\end{array}$ & $\begin{array}{l}\text { Discutir métodos alternativos de comunicação com o } \\
\text { paciente e familiares; } \\
\text { Encorajar a família a incentivar a comunicação do } \\
\text { paciente, seja por gestos, sinais ou escrita }\end{array}$ & $\begin{array}{l}\text { O paciente deverá: } \\
\text {-demonstrar melhor } \\
\text { capacidade de comunicação. }\end{array}$ \\
\hline $\begin{array}{l}\text { Processo familiar } \\
\text { alterado }\end{array}$ & $\begin{array}{l}\text { Encaminhar o paciente e familiares para grupos de apoio; } \\
\text { Ajudar os membros da família a aceitar o paciente com o } \\
\text { seu novo estilo de vida. }\end{array}$ & $\begin{array}{l}\text { O paciente deverá: } \\
\text { - retomar o seu papel de um } \\
\text { membro saudável } \\
\text { na família. }\end{array}$ \\
\hline $\begin{array}{l}\text { Interação social } \\
\text { prejudicada }\end{array}$ & $\begin{array}{l}\text { Proporcionar um relacionamento individual sustentador ao } \\
\text { paciente; } \\
\text { Apoiar as defesas saudáveis do paciente; } \\
\text { Em terapia de grupo estimular o desenvolvimento de } \\
\text { relacionamentos entre os membros através da abertura e } \\
\text { sinceridade. }\end{array}$ & $\begin{array}{l}\text { O paciente deverá: } \\
\text { - identificar novos } \\
\text { comportamentos para } \\
\text { promover a socialização } \\
\text { efetiva. }\end{array}$ \\
\hline $\begin{array}{l}\text { Angústia } \\
\text { espiritual }\end{array}$ & $\begin{array}{l}\text { Valorizar os sentimentos do paciente, ouvindo-o sem julgar } \\
\text { e incentivar o paciente e família a participarem de grupos } \\
\text { de apoio ou grupos religiosos; } \\
\text { Solicitar o acompanhamento da psicologia. }\end{array}$ & $\begin{array}{l}\text { O paciente deverá: } \\
\text { - mostrar-se mais conformado } \\
\text { com a situação. }\end{array}$ \\
\hline $\begin{array}{l}\text { Adaptação } \\
\text { prejudicada }\end{array}$ & $\begin{array}{l}\text { Encorajar o paciente a expressar seus sentimentos e } \\
\text { incentivar a participação em grupos de apoio. }\end{array}$ & $\begin{array}{l}\text { O paciente deverá: } \\
\text { - demonstrar adaptação ao } \\
\text { novo estilo de vida. }\end{array}$ \\
\hline $\begin{array}{l}\text { Enfrentamento } \\
\text { individual } \\
\text { prejudicado }\end{array}$ & $\begin{array}{l}\text { Determinar correlação dos sentimentos e sintomas com os } \\
\text { eventos e mudanças da vida; } \\
\text { Discutir soluções alternativas para o enfrentamento da } \\
\text { situação; } \\
\text { Encorajar o indivíduo a procurar auxílio da psicologia. }\end{array}$ & $\begin{array}{l}\text { O paciente deverá; } \\
\text { - demonstrar capacidade para } \\
\text { solucionar seus } \\
\text { problemas. }\end{array}$ \\
\hline $\begin{array}{l}\text { Distúrbio no } \\
\text { padrão do sono }\end{array}$ & $\begin{array}{l}\text { Orientar o paciente a dormir com a cabeceira elevada; } \\
\text { Discutir com o paciente o melhor decúbito para um sono } \\
\text { mais tranquilo. }\end{array}$ & $\begin{array}{l}\text { O paciente deverá: } \\
\text { - verbalizar melhora do } \\
\text { padrão do sono. }\end{array}$ \\
\hline $\begin{array}{l}\text { Alteração } \\
\text { sensorial de } \\
\text { percepção: } \\
\text { olfato } \\
\end{array}$ & $\begin{array}{l}\text { Reforçar a orientação de que a alteração do senso olfativo } \\
\text { é conseqüência da laringectomia. }\end{array}$ & $\begin{array}{l}\text { O paciente deverá: } \\
\text { - compreender a } \\
\text { razão da alteração senso } \\
\text { perceptiva. }\end{array}$ \\
\hline $\begin{array}{l}\text { Baixo auto } \\
\text { estima } \\
\text { situacional }\end{array}$ & $\begin{array}{l}\text { Auxiliar e encorajar o paciente a expressar seus } \\
\text { sentimentos; } \\
\text { Reforçar suas capacidades positivas, e encorajá-lo a } \\
\text { aceitar seus sentimentos; } \\
\text { Refletir junto com o paciente sobre como conviver com a } \\
\text { situação; } \\
\text { Encorajar o paciente a freqüentar a fonoterapia e a } \\
\text { psicoterapia. }\end{array}$ & $\begin{array}{l}\text { O paciente deverá: } \\
\text { - identificar a origem da } \\
\text { ameaça à auto-estima e } \\
\text { trabalhar para superá-la } \\
\text { expressar uma visão positiva } \\
\text { sobre o futuro. }\end{array}$ \\
\hline
\end{tabular}


(continuação Tabela 2)

\begin{tabular}{|c|c|c|}
\hline $\begin{array}{l}\text { Diagnóstico de } \\
\text { enfermagem }\end{array}$ & Intervenções de enfermagem & Resultados esperados \\
\hline $\begin{array}{l}\text { Distúrbio da } \\
\text { imagem corporal }\end{array}$ & $\begin{array}{l}\text { Encorajar o paciente a expressar como se vê, como encara } \\
\text { as mudanças na sua auto imagem; } \\
\text { Encorajar o contato com a família e amigos; } \\
\text { Discutir sobre a dificuldade que outros familiares podem } \\
\text { ter com as mudanças físicas. }\end{array}$ & $\begin{array}{l}\text { O paciente deverá: } \\
\text { - verbalizar e demonstrar a } \\
\text { aceitação da auto imagem } \\
\text { corporal. }\end{array}$ \\
\hline $\begin{array}{l}\text { Déficit de } \\
\text { conhecimento } \\
\text { sobre o } \\
\text { procedimento } \\
\text { cirúrgico }\end{array}$ & $\begin{array}{l}\text { Ensinar, por meio de folhetos ilustrativos, o procedimento } \\
\text { cirúrgico realizado e as conseqüências da laringectomia; } \\
\text { Reforçar as orientações sobre o autocuidado com o novo } \\
\text { nariz, que é a traqueotomia. }\end{array}$ & $\begin{array}{l}\text { O paciente deverá: } \\
\text { - demonstrar compreensão } \\
\text { sobre o procedimento cirúrgico } \\
\text { realizado e suas } \\
\text { conseqüências. }\end{array}$ \\
\hline $\begin{array}{l}\text { Dor na região } \\
\text { cervical }\end{array}$ & $\begin{array}{l}\text { Avaliar a intensidade da dor antes da administração de } \\
\text { analgésico prescrito pelo médico; } \\
\text { Explicar ao paciente a causa da dor; } \\
\text { Comunicar o médico se a dor persistir após a medicação. }\end{array}$ & $\begin{array}{l}\text { O paciente deverá: } \\
\text { - verbalizar alivio ou } \\
\text { diminuição da dor. }\end{array}$ \\
\hline
\end{tabular}

\section{CONCLUSÕES}

Este estudo nos permitiu entender que o diagnóstico de enfermagem é o ponto de partida para o planejamento da assistência. Embora este trabalho represente um estudo preliminar no levantamento de possíveis diagnósticos de enfermagem voltados para a assistência ao paciente submetido à laringectomia total no período pós-operatório tardio, entendemos que o diagnóstico de enfermagem faz parte de um processo assistencial que deve ser utilizado para direcionar as ações de enfermagem, quer seja durante a internação hospitalar como também durante 0 seguimento ambulatorial.

Este estudo nos mostrou que a assistência ao laringectomizado deve ser voltada não só para a dimensão biológica mas também para o social e o psicológico, pois é um paciente que retorna ao convívio familiar com bruscas alterações de estilo de vida, e muitas vezes altera também todo o contexto familiar.

É importante enfatizar ainda, que há necessidade de outros estudos sobre 0 assunto a fim de subsidiar a assistência de enfermagem efetiva e fundamentada cientificamente.

\section{REFERÊNCIAS BIBLIOGRÁFICAS}

1. Instituto $\mathrm{N}$ acional de $\mathrm{Cancer} \mathrm{(Brasil).} \mathrm{Tipos} \mathrm{de}$ câncer: câncer delaringe [online]. D isponível em:http://inca.org.br/cancer/tipos/ laringe.html.

2. Oliveira BV, O liveira M BM . Reabilitação vocal. In: Brandão LG, Ferraz AR. C irurgia de cabeça e pescoço: princípios técnicos e terapêuticos. São Paulo: Roca; 1989. p. 41320.

3. Kowal ski LP. Câncer de cabeça epescoço: estado atual e perspectivas futuras. Âmb $\mathrm{H}$ osp 1994;5:13-25.

4. Black JM , M atassarin-J acobs E. L uckman \& Sorensen enfermagem médico-cirúrgica: uma abordagem psicofisiológica. 4a ed. Rio de Janeiro: Guanabara K oogan; 1996. p. 42-67.

5. Zago M M F. Plano deensino parao preparo da alta médica do paciente laringectomizado [dissertação]. Ribeirão Preto (SP): U niversidade deSão Paulo; 1990.

6. Zago M M F, Stoppa M JR, M artinez EL. 0 significado cultural deser laringectomizado. Rev Bras C ancerol 1998;44(2):139-45.

7. Christensen PJ, Kenney JW. N ursing process: application of conceptual models. 4a ed. St. Louis: M osby; 1995.

8. Fayram ES, Christensen PJ. Planning: strategies and nursing orders. In: Christensen PJ, Kenney JW. N ursing process: aplication of conceptual models. 4a ed. St. Louis: M osby; 1995. p.165-98.

9. Rossi LA. 0 processo de enfermagem em uma unidade de queimados: análisee reformulação fundamentadas na pedagogia da problematização [dissertação]. Ribeirão Preto (SP): U niversidadedeSão Paulo; 1992.

10. Jesus CAC. Evolução histórica do diagnóstico de enfermagem e sua aplicabilidade no planejamento da assistência. Rev Saúde 1995;6(1/2):37-40.

11. N orth American N ursing D iagnosisAssociation (N AN D A). N ursing diagnosis definitions and classification (1999-2000). Porto Alegre: Artes M édicas Sul; 2000.

12. Risner PB. D iagnosis: analysis and synthesis of data. In: C hristensen PJ , K enney JW. N ursing 
process: application of conceptual models. 3a ed. St. Louis: M osby; 1995. p. 132-157.

13. D oenges M E, M oorhouse M E. N ursing diagnosis with interventions. Philadelphia: F.A. D avis; 1991.

14. Carpenito LJ. D iagnósticos de enfermagem: aplicação à prática clínica. Porto Al egre: Artes M édicas; 1997.

15. Smeltzer SC, Bare BG . Brunner \& Suddarth: tratado deenfermagem médico-cirúrgica. 8a ed.
Rio deJ aneiro: G uanabara Koogan; 1998.

16. O liveira VB, Ramos GA, Dias LAN, Rosoni $M D$. Avaliação nutricional ecomplicações pósoperatórias em pacientes portadores de carcinoma de cabeça e pescoço. Acta 0 ncol Bras 1994;14(4):159-66.

17. White NE, Rither JM , Fry C. C oping, social support, and adaptation to chronicillness. West J N urs Res 1992;14(2):211-4. 